\title{
Corela
}

Cognition, représentation, langage

15-1 | 2017

Vol. $15, \mathrm{n}^{\circ} 1$

\section{Le méta-contributionnel comme forme spécifique de méta-discursivité}

\section{Camille Létang}

\section{OpenEdition}

\section{Journals}

Édition électronique

URL : http://journals.openedition.org/corela/4910

DOI : $10.4000 /$ corela.4910

ISSN : $1638-573 \mathrm{X}$

\section{Éditeur}

Cercle linguistique du Centre et de I'Ouest - CerLICO

Référence électronique

Camille Létang, «Le méta-contributionnel comme forme spécifique de méta-discursivité », Corela [En ligne], 15-1 | 2017, mis en ligne le 26 juin 2017, consulté le 02 mai 2019. URL : http:// journals.openedition.org/corela/4910 ; DOI : 10.4000/corela.4910

Ce document a été généré automatiquement le 2 mai 2019.

\section{(c) (i) (2)(2)}

Corela - cognition, représentation, langage est mis à disposition selon les termes de la licence Creative Commons Attribution - Pas d'Utilisation Commerciale - Partage dans les Mêmes Conditions 4.0 International. 


\title{
Le méta-contributionnel comme forme spécifique de méta- discursivité
}

\author{
Camille Létang
}

\section{Introduction}

1 Appréhender l'omniprésence des commentaires méta-contributionnels comme forme spécifique (et trop peu étudiée) de métadiscursivité présente un intérêt pour la pragmatique de l'interaction. Il est nécessaire par conséquent de considérer l'étude des séquences contributionnelles comme avancée majeure dans les recherches en analyse de la conversation, offrant ainsi une triple réflexion qui impose d'avoir conscience de :

- devoir distinguer le niveau contributionnel du niveau de l'énoncé, dont la différence est marquée par la complétude ;

3 - l'importance du rôle de la ratification expliquant l'existence des séquences contributionnelles et le déroulement des interactions contenant des commentaires métacontributionnels;

4 - la valeur prosodique associée à ces commentaires ou aux contributions, déterminant l'orientation argumentative pour chaque séquence.

5 Les recherches - notamment lors du projet DIASEMIE ${ }^{1}$ - faites jusque ici (et toujours en cours) mènent à la problématique suivante : comment montrer que l'aspect prosodique des contributions présente un enjeu significatif dans l'interprétation et dans la construction des échanges dialogaux, sachant que chaque locuteur laisse des traces de toute forme de commentaire sur son propre énoncé?

6 Nous pouvons d'ores et déjà présenter le schéma du déroulement d'une séquence contributionnelle, prenant en compte la pragmatique, la sémantique, et l'analyse conversationnelle : 
1 feed-back mais

plusieurs réactions possibles conversationnelles (et spécifiquement la maxime de quantité), se trouve introduite à deux reprises la notion de contribution.

"Make your contribution as informative as required.»

« Don't make your contribution more informative than is required. $»^{3}$

en fonction de ce qui est dit en fonction de comment c'est dit

ne va pas de soi - sens social

enchainement clair et fluide

incompréhensions, différentes interprétations

Nous prendrons comme point de départ la distinction entre contribution et énoncé pour nous amener rapidement à l'explication des séquences contributionnelles, pour pouvoir décortiquer ce qui les compose. Nous verrons de ce fait que l'aspect métalinguistique, prosodique, et ratificationnel tient un rôle déterminant dans la construction des échanges dialogaux.

\section{Confusion contribution / énoncé depuis P. Grice}

et ceci sans qu'ensuite aucune référence ne soit faite: Borderieux (2013) à la suite de Nemo (1999) indique ainsi que "la notion de contribution semble familière aux linguistes. [...] Mais à aucun moment de son essai Logic and Conversation le philosophe du langage ne s'attache à définir ce qu'il nomme une « contribution ».

16 A partir de là commence à s'installer une confusion systématique entre "énoncé » et « contribution », les maximes contributionnelles étant conçues comme ayant vocation à expliquer les énoncés. Confusion qui ne sera finalement relevée que près de 25 ans plus tard. A partir de 1999 en effet, se trouve posée par Nemo la question de la co-existence de deux niveaux distincts de contraintes pragmatiques, avec d'une part des contraintes propres aux énoncés et d'autre part des contraintes contributionnelles. Nous pouvons ainsi souligner qu'il existe bien une différence entre le niveau contributionnel et le niveau de l'énoncé, tout comme il est admis de ne pas confondre le niveau de l'intervention et acte de langage.

\section{Qu'est-ce qu'une contribution?}

17 Il est possible en première approximation d'adopter une définition générale dans une optique d'analyse de la conversation, qui désigne une contribution comme la participation d'un locuteur à une conversation, et spécifiquement comme ce que chacun dit à propos de ce qui doit être pris en compte par tous et par exemple sur ce qui a été dit antérieurement (au sein d'une même discussion).

Cette définition préalable de l'objet «contribution» implique ensuite de prendre en compte pleinement la phase de validation/ratification de la demande de prise en compte 
de quelque chose que constitue la contribution, et il devient ensuite indispensable de se donner comme objet d'étude minimale l'ensemble de la séquence contributionnelle ainsi que les nombreuses formes de commentaires métalinguistiques que celle-ci comporte, y compris et sur tous les éléments de la séquence, en matière prosodique. Tout en mettant en relation cet objet pragmatique avec des définitions plus générales du dialogisme.

L'activité sociale de communiquer par le langage, est co-déterminée par tout un ensemble de relations dialogiques, nous dit A. O Barry (2002). Il reprend la citation de Bakhtine (1977 : 136) pour expliquer que « le dialogue, au sens étroit du terme, ne constitue, bien entendu, qu'une des formes, des plus importantes il est vrai, de l'interaction verbale. Mais on peut comprendre le mot "dialogue" dans un sens élargi, c'est-à-dire non seulement comme l'échange à haute voix et impliquant des individus placés face à face, mais tout échange verbal, de quelque type qu'il soit». Dans la théorie de Bakhtine, «la problématique du dialogue implique que tout discours, quelle qu'en soit la nature, se présente comme une reprise-modification, consciente ou pas, de discours antérieurs. Ces relations interdiscursives résultent $\mathrm{du}$ fait que toute forme de conscience ou de connaissance passe par l'activité discursive, de sorte que chaque discours répond à quelque chose, il réfute, il confirme, il anticipe sur les réponses et les objections potentielles, cherche un soutien (...).

Nous voyons par cette introduction de Bakhtine que la notion de contribution semblait être soulevée sans qu'elle n'apparaisse clairement. Les travaux sur l'analyse du discours consacraient peu d'intérêt alors au phénomène de retour sur ce qui a été dit. Sans rejeter ou nier ce qui a été découvert jusque-là, la complexité de cette thèse serait liée à un manque à combler, des explications à compléter, et des observations nouvelles à apporter, afin de comprendre plus exactement le rôle et l'impact des énoncés au niveau méta-contributionnel. En constatant d'emblée que, au cours d'une conversation, les interlocuteurs échangent des propos (contributions) en tentant d'attirer l'attention de l'autre sur ce qui doit être pris en compte.

\section{Contrôle attentionnel}

21 A partir de 1999, Nemo signale la confusion chez Grice et les post-Gricéens des notions de contribution et d'énoncé, et démontre d'une part que les contributions sont typiquement des ensembles d'énoncés, même si cet ensemble est parfois réduit à un seul énoncé (réalité qui est à l'origine de la confusion entre les deux niveaux), et d'autre part qu'elles reposent sur un mécanisme attentionnel fondamentalement important à prendre en compte en analyse du discours.

Pour bien comprendre la réalité des conflits relatifs à la définition collective de ce qui doit être pris en compte, il importe avant tout de comprendre que tout discours implique une focalisation sur quelque chose, et peut donner lieu à polémique même si personne ne conteste en quoi que ce soit la vérité de ce qui est dit.

\section{Conflits à propos de la délimitation du champ attentionnel}

Le champ attentionnel est assez largement, du fait du processus de ratification, un champ de bataille. Car si l'ensemble de ce qui mérite d'être pris en compte, autrement dit le champ attentionnel au sens large, est défini par l'ensemble des contributions des 
interlocuteurs à la définition de celui-ci, la prise en compte effective de chacun des éléments qui le compose n'est pas acquise, le fait qu'il le soit ou non devenant lui aussi objet de conflit.

S'agissant du premier aspect de la question, à savoir la participation à la définition du champ attentionnel elle-même, il faut noter qu'il n'est pas possible de supposer qu'il résulterait d'un simple principe pragmatique général prévoyant la possibilité et le devoir de contribuer à une question en cours que dans une conversation ou un débat le droit de contribuer soit effectif et équitable. Des conflits peuvent éclater, et nous pourrons ainsi observer la mise en place dans les domaines les plus sensibles d'un ensemble de normes collectives visant à assurer une certaine égalité dans l'interlocution. Des formes de réglementation des tours contributionnels sont ainsi mises en place ${ }^{4}$, qui peuvent être complètement formalisées dans des domaines comme le domaine juridique ou politique.

S'agissant du second aspect de la question, le problème prend un ensemble de formes, puisqu'il concerne autant le fait d'ignorer délibérément tel ou tel élément que le fait de le refuser ou de le minimiser, mais aussi celui de contester le droit même de vouloir attirer l'attention sur quelque chose, notamment quand il s'agit de détourner l'attention d'autre chose.

\section{La parole comme demande de prise en compte de quelque chose}

Ce mécanisme, que les psychologues nomment attention contrôlée (modèle de Engle et al. 1999), peut se traduire par le fait que très souvent parler revient à « attirer l'attention de quelqu'un sur quelque chose en lui demandant de le prendre en compte» (Nemo, 1992). Cela ne peut pas être fait de façon neutre, car on ne peut pas attirer l'attention de quelqu'un sur quelque chose sans lui indiquer d'une façon ou d'une autre comment cette chose doit être prise en compte (Nemo, 1992), ce que les psychologues appellent cette fois « référencement social».

\section{Construction d'un champ attentionnel partagé}

27 Si la parole peut-être vue comme une demande de prise en compte de quelque chose, c'est parce qu'on ne peut pas attirer l'attention de quelqu'un sur quelque chose sans que cette personne ne suppose les raisons pour lesquelles on la sollicite. Ainsi, cela ne peut pas être fait de façon neutre et on ne peut pas attirer l'attention de quelqu'un sur quelque chose sans lui indiquer d'une façon ou d'une autre comment il doit considérer cette chose. A partir de là, il est possible de poser que les échanges conversationnels reposent sur l'existence d'un champ attentionnel partagé et sur un principe de présomption de contribution qui à la suite de Nemo (1999), et Nemo, Létang, Petit (2016) peut être formulé ainsi :

Sachant que l'objet de l'échange est la co-définition par les participants à l'échange à la fois de l'ensemble de ce qui doit être pris en compte (ou non) à propos de quelque chose et de la façon dont cela doit être pris en compte, chaque intervention est présumée être une contribution à cet objectif collectif.

Trois choses à ce stade méritent d'être soulignées :

29

- parler de co-définition de ce qui doit être pris en compte implique par définition que les participants peuvent accepter ou non la demande de prise en compte de quelque chose, 
ainsi que discuter le degré de prise en compte, autrement dit implique un processus de ratification.

\begin{abstract}
présuppose un champ attentionnel co-construit, une présomption de contribution et un
\end{abstract} processus de ratification.

31 - il est possible ensuite d'observer empiriquement que les échanges argumentatifs sont soumis à des contraintes contributionnelles qui s'avèrent souvent très proches (e.g. complétude) des contraintes contributionnelles classiques comme les maximes de quantité, de qualité ou de pertinence, à ceci près que l'enjeu de la contribution n'est pas informationnelle mais est de définir ce qui doit être pris en compte et de la façon dont cela doit être pris en compte.

\title{
4. Contribution et scène interlocutive
}

Les travaux en analyse du discours (entre autres, Kerbrat-Orecchioni 2005) ont démontré que lors d'une discussion, chaque participant tente à la fois de faciliter la compréhension de ce qui est dit par l'interlocuteur, et d'aller vers un consensus. Partant de ce constat, nous avons pu aussi voir dans nos recherches que les échanges sont soumis à des contraintes contributionnelles qui s'avèrent souvent très proches des contraintes contributionnelles classiques comme la maxime de quantité, de qualité ou de pertinence complétude (pour reprendre le terme de Portugues 2011) - à ceci près que l'enjeu de la contribution n'est pas informationnel mais est de définir ce qui doit être pris en compte et la façon dont cela doit être pris en compte.

Par ailleurs, nous pouvons tenir pour acquis dans ce qui suit que :

- toute contribution est indissociable de la séquence contributionnelle dont elle fait partie et qui inclut la ratification de la contribution;

- les interlocuteurs peuvent ne pas tomber d'accord sur ce qui doit être pris en compte (ou pas), sur l'importance relative de tout ce qui doit être pris en compte ou sur la façon de le prendre en compte ;

- est associé à tout échange (argumentatif) une mémoire des réussites et des échecs du processus de co-définition de ce qui doit être pris en compte et de sa dynamique, que nous nommons scène interlocutive;

- toute contribution est amenée à anticiper la contrainte de ratification et certains moyens de le faire sont des moyens linguistiques ;

- le processus de ratification lui aussi repose sur des formes linguistiques.

\section{Le métalinguistique}

Toute étude du rôle des contributions en contexte dialogal comme celle que nous avons menée conduit à être confronté à l'omniprésence des commentaires métalinguistiques. Nos observations nous ont amené à penser que si le méta-contributionnel est une particularité du méta-discursif, c'est parce qu'il englobe plusieurs aspects issus du métalinguistique tels que le commentaire implicite ou la remarque masquée, dévoilant l'intention du locuteur.

35 Comme l'explique J. Rey-Debove (1997), «il est généralement admis que le langage possède une "fonction métalinguistique", en d'autres termes, que le langage est parfois

Corela, 15-1 | 2017 
employé pour parler du langage. Le discours du linguiste en est le meilleur exemple, par la permanence du thème; mais chacun de nous, quel que soit son propos, recourt nécessairement à cette "fonction métalinguistique" lorsque des commentaires sur le dire sont indispensables à la communication, notamment lorsqu'il faut ajuster le discours à ce qu'il est censé désigner et signifier (pour rappeler, pour apprendre, pour désambiguïser). »

\section{Le métadiscours}

Il y a dans les séquences contributionnelles ce que G-E Sarfati (1997) nomme des «opérations métadiscursives (dire et redire). C'est une qualité distinctive des langues naturelles que de permettre aux énonciateurs de commenter leur propre discours ».

Une recension des types fonctionnels du métadiscours a été proposé par M.-M de Gaulmyn (1987, 170), qui suggère de distinguer entre trois sortes de formulations métalangagières : les énoncés métalinguistiques à proprement parler (qui ont pour objet la langue elle-même), les énoncés métadiscursifs (qui s'appliquent directement aux paroles tenues), les énoncés métacommunicationnels enfin (qui portent sur le déroulement de l'échange). Aussi, par ailleurs, A. Steuckardt et A. Niklas-Salminen (2005) ont constaté qu'« il y a deux fonctions des marqueurs formés sur le dire : le locuteur s'en sert tantôt pour inscrire le discours d'autrui dans son discours, tantôt pour pouvoir revenir sur son propre discours.»

\section{Emploi des expressions méta-contributionnelles et expressions méta-discursives}

Par le biais d'émissions télévisées, radiophoniques, ou simplement entendus par hasard dans des conversations, nous avons relevé divers énoncés qualifiés par nous-mêmes d'expressions méta-contributionnelles. Dans un premier temps, nous avons remarqué que ces expressions servent à « recadrer » la conversation, à expliciter pourquoi tel locuteur souhaite attirer l'attention sur tel point en particulier, souligner quelque chose, ne pas laisser de place à l'ambiguïté. Celui qui emploie ce type d'expressions explique ce qu'il est en train de faire tout en le disant. Dans un second temps, nous avons également distingué un caractère de méta-discursivité, c'est-à-dire qui renvoie à ce qui est dit à propos de ce qui est dit. Il s'agit d'un commentaire implicite mais "inclus» dans l'énoncé que fait le locuteur afin d'anticiper une réaction, ou d'exposer son point de vue. Ces expressions, dans tous les cas, nous montrent que dans la scène interlocutive que créent les interactants, les séquences contributionnelles sont formées sur des enchainements ratificationnels parfois présentés par les locuteurs comme un retour sur ce qui a été dit, ou une réaction directe, agrémenté d'un commentaire -métalinguistique - explicatif de cette contribution.

Une liste non exhaustive des exemples relevés est présentée dans ce tableau : 


\begin{tabular}{|c|c|c|c|c|c|c|}
\hline expressions & sources $^{5}$ & $\begin{array}{l}\text { métalangage } \\
\text { indiquant } \\
\text { que le } \\
\text { locuteur } \\
\text { veut revenir } \\
\text { sur quelque } \\
\text { chose, ou } \\
\text { modifier } \\
\text { quelque } \\
\text { chose, ou } \\
\text { commenter }\end{array}$ & $\begin{array}{l}\text { notes / } \\
\text { remarques }\end{array}$ & $\begin{array}{l}\text { référence } \\
\text { aux } \\
\text { maximes } \\
\text { conver- } \\
\text { sationnelles } \\
\text { de Grice } \\
\text { (laquelle?) }\end{array}$ & $\begin{array}{l}\text { valeur } \\
\text { ratification- } \\
\text { nelle (+ ou - } \\
\text { forte) }\end{array}$ & \begin{tabular}{|l} 
impact \\
prosodi- \\
que \\
(oui/ \\
non)
\end{tabular} \\
\hline $\begin{array}{l}\text { est-ce } \\
\text { qu'on } \\
\text { n'aurait } \\
\text { pas fait le } \\
\text { tour de la } \\
\text { question }\end{array}$ & $\begin{array}{lcc}\text { CNTRL } & - & \text { asso. } \\
\text { BOI_M1_09.txt } & \end{array}$ & commenter & $\begin{array}{l}\text { tentative de } \\
\text { clore un } \\
\text { sujet }\end{array}$ & de quantité & forte & $\begin{array}{l}\text { oui } \\
\text { fatigue? }\end{array}$ \\
\hline $\begin{array}{l}\text { mais là je } \\
\text { fais une } \\
\text { parenthèse } \\
\text { juste sur la } \\
\text { question de } \\
\text { la com }\end{array}$ & & commenter & $\begin{array}{ll}\text { situer } & \text { le } \\
\text { propos, } & \text { le } \\
\text { contexte } & \end{array}$ & de manière & assez forte & non \\
\hline $\begin{array}{l}\text { là-dessus } \\
\text { ben je vais } \\
\text { finir }\end{array}$ & $\begin{array}{l}\text { CNTRL } \\
\text { Assemblee_MAU_08.txt }\end{array}$ & commenter & $\begin{array}{ll}\text { situer } & \text { le } \\
\text { propos, } & \text { le } \\
\text { contexte } & \end{array}$ & de manière & assez forte & non \\
\hline $\begin{array}{l}\text { il y aurait } \\
\text { beaucoup à } \\
\text { redire }\end{array}$ & $\begin{array}{l}\text { entendu dans des } \\
\text { conversa-tions }\end{array}$ & commenter & $\begin{array}{l}\text { dire sans le } \\
\text { dire }\end{array}$ & de quantité & moyenne & $\begin{array}{l}\text { ironie? } \\
\text { agacé ? }\end{array}$ \\
\hline $\begin{array}{l}\text { il n'a pas } \\
\text { eu un mot } \\
\text { pour Jean, } \\
\text { alors qu'il } \\
\text { avait tout } \\
\text { préparé }\end{array}$ & & commenter & constater & de quantité & nulle & $\begin{array}{l}\text { déçu? } \\
\text { étonné? }\end{array}$ \\
\hline $\begin{array}{l}\text { en fait avec } \\
\text { Titi on s'est } \\
\text { dit, pour en } \\
\text { revenir aux } \\
\text { courses, } \\
\text { qu'on les } \\
\text { ferait } \\
\text { l'après- } \\
\text { midi }\end{array}$ & & revenir sur & $\begin{array}{l}\text { on s'éloigne } \\
\text { du sujet }\end{array}$ & de relation & moyenne & non \\
\hline
\end{tabular}




\begin{tabular}{|c|c|c|c|c|c|c|}
\hline $\begin{array}{l}\text { oui on avait } \\
\text { dit qu'on } \\
\text { partirait } \\
\text { que deux } \\
\text { semaines } \\
\text { mais [prêt } \\
\text { à se faire } \\
\text { couper la } \\
\text { parole] est- } \\
\text { ce que je } \\
\text { peux } \\
\text { revenir sur } \\
\text { mes } \\
\text { propos? Si } \\
\text { on part } \\
\text { trois semaines } \\
\text { moi je } \\
\text { préfèrerais }\end{array}$ & & modifier & $\begin{array}{l}\text { demande de } \\
\text { rectification }\end{array}$ & de manière & assez forte & agacé ? \\
\hline $\begin{array}{l}\text { tu m'en } \\
\text { diras tant }\end{array}$ & & commenter & constater & $\begin{array}{l}\text { de quantité } \\
\text { ou de } \\
\text { qualité }\end{array}$ & forte & $\begin{array}{l}\text { sarcas- } \\
\text { me? } \\
\text { ironie? } \\
\text { moque- } \\
\text { rie? }\end{array}$ \\
\hline $\begin{array}{l}\text { vous allez } \\
\text { me dire }\end{array}$ & & commenter & anticipation & de quantité & forte & non \\
\hline $\begin{array}{l}\text { on peut } \\
\text { dire ça } \\
\text { comme ça }\end{array}$ & & commenter & $\begin{array}{l}\text { être } \\
\text { faussement } \\
\text { d'accord }\end{array}$ & de qualité & moyenne & $\begin{array}{l}\text { ironie? } \\
\text { approba- } \\
\text { teur? }\end{array}$ \\
\hline $\begin{array}{lr}\text { j'suis aux } \\
35 \text { heures } \\
\text { dès le } \\
\text { mardi soir, } \\
\text { déjà. Pour } \\
\text { tout vous } \\
\text { dire. }\end{array}$ & $\begin{array}{l}\text { radio France Bleu - } \\
\text { émission "on se dit } \\
\text { tout" - thème : ma } \\
\text { passion, ma vie }\end{array}$ & commenter & $\begin{array}{l}\text { tout a été } \\
\text { dit }\end{array}$ & de quantité & moyenne & non \\
\hline
\end{tabular}

\section{La prosodie}

Les travaux menés sur le profilage prosodique des énoncés depuis quelques années ont démontré que faisait partie de ce qui est réellement dit toutes les formes de commentaire prosodique associées à la phrase dite (Nemo, Létang, Petit, 2016). Ils ont en particulier montré que ces commentaires avaient pour objet de définir l'orientation contributionnelle de ce qui est dit, et il a été spécifiquement démontré que les 
commentaires prosodiques en question concernaient :

- L'orientation argumentative associée à un mot donné. nous observons d'emblée que cette ratification apparaît fortement modulable, notamment d'un point de vue argumentatif. Nous observons aussi que le oui peut être convaincu ou non, et qu'il est parfois plus une marque de politesse que de ratification, ou associé à des formes variées de réticence ou de réserve, et enfin qu'il peut même parfaitement vouloir dire non. La prosodie peut marquer une forme d'interaction complexe. Nous pouvons entendre dans les « oui » des jeux polyphoniques indiquant la position du locuteur, de l'interlocuteur, et la position que le locuteur prend par rapport à l'interlocuteur. La pragmatique est intégrée dans le message linguistique d'emblée (« what is said?»). Et par exemple pour «quelques» en français - décrit par Ducrot (1973) comme orienté vers une quantité élevée alors que les dictionnaires le décrivent eux comme orienté vers une quantité faible - nous pouvons également constater que l'orientation argumentative dépend du commentaire prosodique associé à la réalisation $\mathrm{du}$ «quelques ». De même, si nous prenons un enchaînement argumentatif décrit depuis Ducrot comme anti-orienté, tel que :

«Il a lu quelques livres de Balzac mais il y a longtemps »

nous constatons que très souvent la prosodie de « il a lu quelques livres» va créer une coorientation avec le second énoncé (« mais il y a longtemps »).

Les commentaires prosodiques peuvent donc inverser une orientation argumentative.

La prosodie enrichit les modèles portant sur les contraintes du dire, car elle révèle une dimension polyphonique qui permet d'interpréter de manière complexe et complète un énoncé. Cela donne un type d'orientation argumentative encore jamais étudié à ce jour.

Le ton qu'on emploie est un commentaire sur ce qui est dit. 
49 il suscite un comportement chez son interlocuteur. Celui-ci va devoir prendre en compte la façon dont lui a été transmis le message pour moduler le sens à ce qui a été dit. L'interlocuteur se basera sur cette prosodie pour enchainer (humour, ironie, etc.)

Que ce soit en étudiant les « oui » ou les expressions méta-contributionnelles, nous avons pu avancer l'idée que la ratification a un lien avec la prosodie dans le sens où l'interlocuteur peut choisir de se baser (pour enchainer) sur ce qui est dit (les mots, le sens propre) ou sur la façon dont cela a été dit (interprétation des sous-entendus dans l'intonation). Le commentaire sur le «oui » intoné ou n'importe quelle contribution entraine une réaction de la part de l'interlocuteur.

\section{Métalinguistique et ratification}

51 Si la prosodie a un rôle déterminant dans la compréhension d'un énoncé, c'est parce qu'elle permet à l'interlocuteur d'avoir des repères pour la suite (l'enchainement) des énoncés à fournir. En fait, nous ne nous basons pas uniquement sur les mots employés par notre interlocuteur pour lui répondre, nous rebondissons également (voire parfois uniquement) sur la prosodie. Nous pouvons ainsi envisager qu'il puisse exister parfois une sorte de décalage -plus ou moins grand- entre ce qui est dit et ce " qui est pensé ». Par exemple, dire oui quand on pense non, ça s'entend! Et l'interlocuteur peut légitimement rétorquer : "oh bah puisque que tu le prends comme ça... ", équivalant ainsi à une réponse anti-orientée à l'énoncé précédent (« oui »). Les contraintes prosodiques sont donc des contraintes linguistiques à part entière pour l'interprétation des énoncés.

52 Nous réalisons que, très souvent, la forme prosodique fournit une représentation de la scène interlocutive, car elle permet d'avoir accès non seulement à la position du locuteur, mais aussi à la position supposée de l'interlocuteur par rapport à celle-ci, et même à la position du locuteur par rapport à la position que l'interlocuteur a vis-à-vis de sa position initiale.

53 Toute contribution peut a priori être :

- rejetée (comme hors de propos ou inacceptable);

- ignorée (comme hors de propos ou inacceptable);

- ratifiée tacitement par un silence ;

- ratifiée comme secondaire ou marginale, autrement dit comme méritant peu d'attention ;

- ratifiée comme importante, et donc comme méritant d'être prise en compte avec toute l'attention nécessaire.

Sur cette base, nous appelons "ratification" la forme de feed-back que reçoit toute contribution et en fonction de laquelle la demande de prise en compte de quelque chose est intégrée ou non au « consensus interlocutif ».

Et nous appelons scène interlocutive la forme de common ground qui associe pour tout participant l'ensemble de ce qui fait dissensus et de ce qui fait consensus. Nous admettons enfin que puisque les contributions reposent sur une demande de prise en compte de quelque chose, et que cette demande peut être satisfaite ou non, toute contribution donnera lieu à une séquence ratificationnelle comportant un nombre minimal d'étapes.

Ces étapes sont : 
une phase contributionnelle ;

une phase de réaction à ce qui a été dit ;

une phase d'ajustement interlocutif ;

une phase finale d'intégration de tout ou partie de la contribution initiale au consensus interlocutif en tant que conclusion du dialogue.

Ce qui revient à dire que les contributions ne peuvent être décrites en dehors des formes de retour, de reprise ou de silence qu'elles engendrent inévitablement.

Ce sont toutes ces formes de feed-back contributionnel que nous nommons ici ratification, en notant que la séquence ratificationnelle complète peut être longue, dès lors que la ratification elle-même est soumise à ratification, etc.

Toute étude empirique du processus de (non) ratification, qu'elle soit menée sur des données orales (e.g. débat) ou écrites (e.g. fils de commentaires), commence par un repérage sur corpus de tous les éléments ou séquences linguistiques (.e.g. lexicaux, discursifs, prosodiques) qui portent sur des contributions antérieures et sont susceptibles de définir la valeur contributionnelle. A l'intérieur de cet ensemble, si nous isolons ensuite l'ensemble des commentaires méta-contributionnels plus ou moins explicites, nous observons des formes de récurrence ou même de conventionnalisation de certains éléments, mots ou expressions. Nous pouvons ensuite continuer dans ce dernier ensemble à séparer les éléments en quelque sorte spécialisés dans l'expression de tels commentaires méta-contributionnels, des formes plus polyvalentes et moins spécialisées. Nous sommes alors capables de nommer marques lexicalisées de ratification contributionnelle (MLRC) cette classe d'expressions méta-communicationnelles. Nous obtenons de ce fait non seulement des listes de telles expressions, mais pour chacune d'entre elles un relevé de la façon dont elles sont employées. Et c'est alors qu'un travail d'analyse plus fouillé peut commencer, relatif notamment à leur éventuelle polyfonctionnalité, mais surtout à la description de leur rôle argumentatif réel dans chaque emploi. Malgré cela ce qu'il faut souligner, c'est qu'une telle étude des MLRC constitue une porte d'entrée unique sur la nature exacte des contraintes contributionnelles ou argumentatives dont elles sont la trace. Pour prendre un exemple concret, nous pouvons considérer un sous-ensemble de MRLC relatif à ce qui est sans doute la contrainte contributionnelle majeure, à savoir la contrainte de complétude (dont la forme gricéenne est la maxime de quantité).

Ce que vous ne dites pas

Vous passez sous silence ...

C'est un peu court

Vous ne dites rien de...

On a fait le tour de la question?

Je vous signale que...

Je n'ai rien à ajouter

Vous semblez ignorer que..

T'en as trop dit ou pas assez

Tu dis ça tu dis rien

C'est bon on a compris

Tout le monde sait ça.

On a entendu ça cent fois...

Vous oubliez de dire

Ne pas oublier de dire ... 


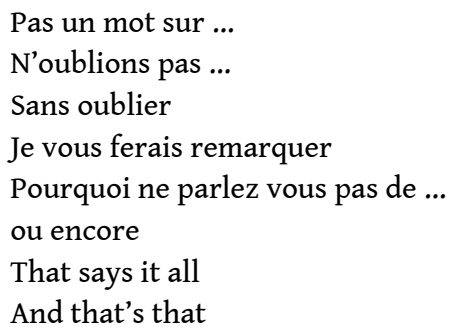

- il est indispensable de mener l'étude des ressorts linguistiques de l'argumentation au niveau des contributions (y compris pour comprendre ce qui se passe au niveau des énoncés) ;

- il y a eu méprise chez Grice non seulement sur le niveau contributionnel (confondu avec le niveau de l'énoncé) mais sur la nature informationnelle (et non argumentative) des contraintes contributionnelles ;

- les contraintes contributionnelles les plus classiques s'avèrent relatives à des enjeux directement argumentatifs, à savoir par exemple la co-définition de l'ensemble de ce qui doit être pris en compte, sachant que les conclusions tirées de l'échange dépendent directement de contenu de cet ensemble ;

- l'étude linguistique et empirique des processus de ratification, tant du point de vue prosodique que lexical, permet d'objectiver des contraintes ou niveaux de contraintes largement inaccessibles à une réflexion non-empirique ;

70 - La prosodie est essentielle à la constitution de la scène interlocutive, elle joue un rôle majeur dans l'enchaînement ; 
- L'aspect méta-contributionnel, vu comme une spécificité de méta-discursivité, permet de cerner, de construire le discours et l'interprétation.

71 La parole, pour n'importe quel locuteur, est une parole publique, c'est-à-dire que tout le monde peut se l'approprier et lui donner un sens, une interprétation. Chaque énoncé est une parole qui nécessite un engagement de la part de l'interlocuteur, qu'il réagisse ou non à ce qui est dit. D'où l'importance de la ratification.

Mais, comme nous l'avons vu, la parole est une contribution, où quelque chose qui est dit va influencer le reste de la conversation (nuancer, rectifier, contredire...).

Parler, c'est toujours attirer l'attention sur quelque chose en demandant à ce que cela soit pris en compte. Et on ne parle jamais pour ne rien dire. Dans un échange oral, l'utilisation du langage par un locuteur n'a pas seulement pour but d'exprimer un contenu, mais d'influer sur ses interlocuteurs. Il y a, dans le dit, le non-dit, et la façon de dire, beaucoup d'informations à relever pour la compréhension d'un énoncé, permettant de se positionner pour enchainer dans la conversation (est-ce qu'on se base sur le contenu ou sur le méta?). Les formes métalinguistiques ont donc un rôle dans la structuration et l'explication des échanges dialogaux.

\section{BIBLIOGRAPHIE}

Authier-Revuz, J. (2004). La représentation du discours autre : un champ multiplement hétérogène, in J.-M. Lopez-Munoz, S. Marnette \& L. Rosier (éds.), Le discours rapporté dans tous ses états : questions de frontières. P. 35-53.

Barry, A. O. (2002). Les bases théoriques en analyse du discours. Documents de la Chaire MCD, 159.

Borderieux, J. (2013). La construction textuelle du brevet d'invention : analyse et théorisation de la strate contributionnelle. Thèse de doctorat, Université d'Orléans.

Clark, H. and Schaefer, E. (1989). Contributing to discourse. Cognitive science, 13, 259-294.

Ducrot, O. (1973). La preuve et le dire, Repères linguistiques.

Engle, R. W., Kane, M. J., \&Tuholski, S. W. (1999). Individual differences in working memory capacity and what they tell us about controlled attention, general fluid intelligence, and functions of the prefrontal cortex. In A.Myake \& P. Shah (Eds.), Models of working memory: Mechanisms of active maintenance and executive control (pp. 102-134). New York: Cambridge University Press; US.

Furberg, M. (1963). Locutionary and Illocutionary Acts: A Main Theme in J.L. Austin's Philosophy, Göteborg: Gotheburg Studies in Philosophy.

Gaulmyn, M. M. D. (1987). Reformulation et planification métadiscursives. Décrire la conversation, 167-198.

Grice, H. P. (1975). Syntax and semantics 3: Speech arts, Cole et al. "Logic and conversation", pp. 41-58.

Kerbrat-Orecchioni, C. (2005). Le discours en interaction, Paris : A. Colin. 
Nemo, F. (1992). Contraintes de pertinence et compétence énonciative : l'image du possible dans l'interlocution. Thèse de l'EHESS. Paris.

Nemo, F. (1999). « The Pragmatics of Signs, the Semantics of Relevance and the Semantic/ Pragmatics Interface", in Ken Turner, The Semantics-Pragmatics Interface from Different points of View, CRiSPI Series, Amsterdam: Elsevier Science, pp 343-417.

Nemo, F. (2006). "The Pragmatics of Common Ground. From Common knowledge to Shared Attention and Social Referencing". In Lexical Markers of Common Grounds (Studies in Pragmatics, Volume 3) A. Fetzer \& K. Fischer (Eds). Amsterdam : Elsevier Science (224 pages). 143-158

Nemo, F., Létang, C., Petit, M. (2016). « Prosodic constraints on argumentation: from individual utterances to argumentative exchanges ». Argumentation and Reasoned Action: Proceedings of the first European Conference on Argumentation. Dima Mohammed and Marcin Lewinski (Eds). Volume I. Collection Studies in Logic and Argumentation, London: College publication. Volume 1. 520-539.

Portugues, Y. (2011). Contraintes pragmatiques de complétude et linguistique des contributions en théorie du texte et de l'organisation textuelle : élaboration d'une heuristique appliquée au roman de formation. Thèse de doctorat, Université d'Orléans.

Rey-Debove, J. (1997). Le métalangage. Paris : Armand Colin.

Sarfati, G-E. (1997). Éléments d'analyse du discours. Paris, Nathan.

Steuckardt, A., \& Niklas-Salminen, A. (2005). Les marqueurs de glose. Langues et langage.

\section{NOTES}

1. Discrimination Automatique des Sens d'Emploi des Mots par l'Intonation. Projet de 2013-2015 mené par F. Nemo à l'Université d'Orléans, pour une étude de la résolution de la polysémie par des caractéristiques prosodiques

2. Grice reprend en fait des principes décrits par Mats Furberg (1963), mais est bien responsable de l'introduction du terme « contribution » que l'on ne trouve pas chez Furberg.

3. Que votre contribution soit aussi informative que nécessaire.

Que votre contribution ne soit pas plus informative que nécessaire.

https://fr.wiktionary.org/wiki/maximes_de_Grice Consulté le 12 mars 2017.

4. On peut citer par exemple le fait que cela soit la défense qui dans un procès ait le dernier mot, cette norme tenant compte du fait qu'aux termes des débats, l'intervention finale a le pouvoir de laisser les participants dans une perspective attentionnelle particulière.

5. http://www.cnrtl.fr/

6. https://sites.google.com/site/diasemie/

7. Non exhaustive, extraits de corpus divers tels que ESLO (enquête sociolinguistique à Orléans), CNRTL (ortolang), ou issus de conversations orales (cantine, réunion, soirées entre amis...) 


\section{RÉSUMÉS}

Cet article introduit les formes de méta-discursivité qui concernent spécifiquement le niveau contributionnel. Après avoir rappelé la façon dont la pragmatique contemporaine (à partir de Grice) a pu longtemps confondre les notions de contribution et d'énoncé, et après avoir présenté rapidement les principales conclusions des travaux qui depuis dix ans ont permis d'isoler et de caractériser les contributions comme ensemble d'énoncés contribuant à la définition du champ attentionnel, il définit et illustre la notion de commentaire méta-contributionnel et métadiscursif en présentant différentes formes de commentaires méta-contributionnels, y compris les commentaires prosodiques, en expliquant comment ils structurent la réalité des échanges dans les dialogues.

This article introduces forms of meta-discourse that specifically concern contribution level. After recalling how the notions of contribution and statement have been confused for a long time by contemporary pragmatics (starting from Grice), we briefly present the main conclusions of more recent works which have made it possible to characterize contributions as a set of utterances which contribute to the definition of the attentional field. We then define and illustrate both meta-contribution in its diverse uses and meta-discursive comments, including prosodic comments. We finally attempt to explain how the latter two notions structure the reality of exchanges in dialogues.

\section{INDEX}

Mots-clés : métadiscours, contributions, énoncés, ratification, prosodie, interprétation.

Keywords : Métadiscours, contribution, utterances, ratification, prosody, interpretation

\section{AUTEUR}

\section{CAMILLE LÉTANG}

camille.letang@univ-orleans.fr

Université d'Orléans

Laboratoire Ligérien de Linguistique, UMR 7270 (CNRS, Orléans, Tours, BNF) 